\title{
Seeing and touching aesthetic objects: II. Descriptions
}

\author{
MARTIN S. LINDAUER \\ State University of New York, College at Brockport, Brockport, New York
}

\begin{abstract}
Lindauer, Stergiou, and Penn (1986) found that affective, sensory, and cognitive judgments of aesthetic objects were similar across visual and tactile modes of presentation. The present study $(N=60)$ attempted to specify the possible locus of equivalence. Descriptions were given to aesthetic and quasi-aesthetic objects which were seen, touched, or both seen and touched. The latency, duration, and frequency of the descriptions (representing input, mediation, and output stages, respectively) were recorded. Evidence of equivalence between the senses was again found on all measures and modes of presentation.
\end{abstract}

Lindauer, Stergiou, and Penn (1986) found more similarities than differences in the sensory, perceptual, and cognitive meanings of ceramic vessels that were presented in the visual, tactile, or combined modes. The ratings, however, did not clarify where equivalence took place. It may have occurred initially, close to the time of input (i.e., during perception), or it may have been achieved at some later stage of processing (i.e., during evaluation and judgment). Another possibility is that equivalence was achieved at the time of responding, that is, when the descriptive terms were rated. In the present study, some of these possibilities were examined.

Subjects described their visual, tactile, and combined visual-tactile experiences of two objects: an aesthetic ceramic vessel (Figure 1) and a quasi-aesthetic ceramic scrap (see Lindauer et al., 1986, Experiments 1 and 3, respectively). Latency, duration, and the number of responses were recorded. The latency measure, closer in time to sensory-perceptual input, reflects early processing. The duration measure, representing mediational processes (i.e., the time needed for thinking), is more cognitive in character than is latency. Frequency of output, an overt and direct measure of the processes involved, mirrors the end of the process.

Consequently, equivalence or nonequivalence on one or more of the three measures clarifies when a reaction to sight and touch occurred. Two different kinds of stimuli were presented in order to determine whether the locus of equivalence depended on the type of object presented.

\section{METHOD}

A maximum of 20 subjects (undergraduate volunteers from a general psychology class) participated in each of three groups: sight, touch, and combined sight and touch groups. The number of subjects varied because of the exclusion of several outliers among the latency ( $\geq 15 \mathrm{sec}$, $N=10$ ) and duration scores ( $\geq 150 \mathrm{sec}, N=5$ ). More women than men

The author's mailing address is: Department of Psychology, State University of New York, College at Brockport, Brockport, NY 14420. participated ( $N=38$ and 22 , respectively), ranging from 10 women in the combined sight-touch condition to 15 women in the visual condition. Each subject was instructed to describe his or her experience of an object that was seen, touched, or both seen and touched, as completely and for as long as he or she could, using any words that came to mind while the object was present. Subjects were alternately presented with either the aesthetic or quasi-aesthetic object first; the two presentations were separated by a $20-30$ min interval, during which time two other unrelated tasks were performed. Subjects were assigned to the seeing, touching, or combined mode of presentation according to the order in which they appeared at the lab.

The time period, clocked by a hand-held Micronta chronograph (in $1 / 100^{-s e c}$ intervals), began when a subject either lifted a screen (blocking his or her view of the object) or placed his or her hands behind it, and ended when a subject reported that he or she was finished. The latency measure ended, and the duration measure began, as soon as a subject began to verbalize his or her experience. The total number of descriptive terms reported, nearly all of which were single words, were counted. (The conditions were unidentified during the count. When several apparent synonyms were reported-an infrequent occurrenceeach was counted as one response.)

\section{RESULTS AND DISCUSSION}

Reaction times for the different senses were not equivalent $[F(2,47)=5.72, p<.01$; Figure 2]: sight was faster than either touch or combined sight and touch (mean $=4.23,6.32$, and $5.47 \mathrm{sec}$, respectively). Only the differ-

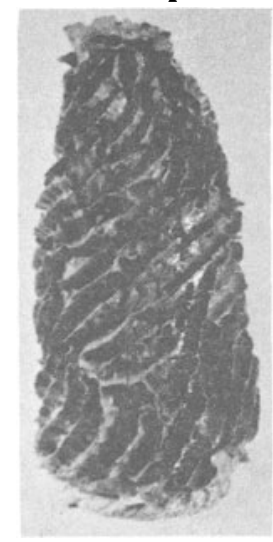

Figure 1. The aesthetic object used. 


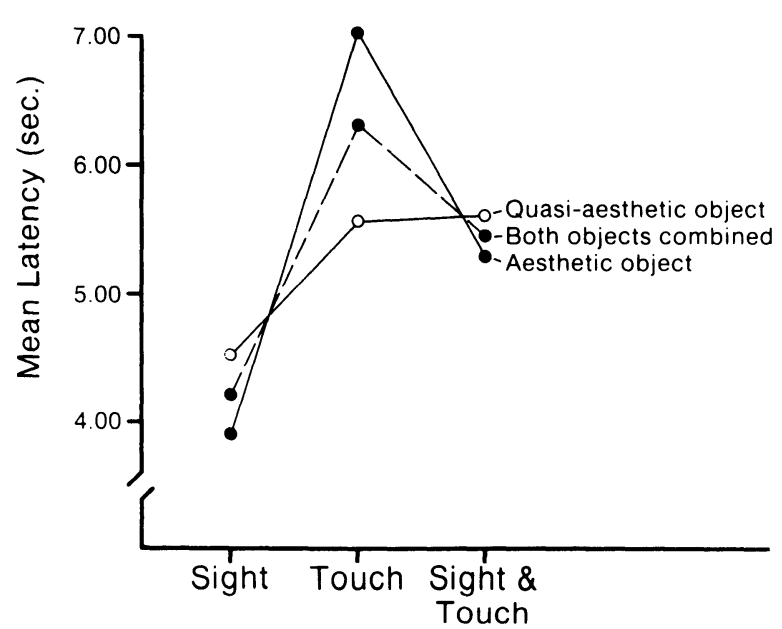

Figure 2. Latency of responses to the aesthetic and quasi-aesthetic object on three sensory modes.

ence between sight and touch, according to a simple effects test, was significant $(p<.05)$. Although the interaction between mode and type of object failed to reach significance $[F(2,47)=2.15, p>.05]$, further analysis did reveal a relationship. (The differences, in part, were masked by the curvilinear nature of the data.) Reaction time for the aesthetic object that was presented in the visual mode (mean $=3.92 \mathrm{sec}$ ) was faster than reaction times in either the tactile or combined modes [mean = 7.04 and 5.30 , respectively; $t(37-38)=1.71-2.45$, $p<.05$, one-tailed test]. Furthermore, the reaction to the tactile presentation of the aesthetic object was slower than was the reaction to the quasi-aesthetic object [mean $=7.04$ and 5.59, respectively: $t(14)=2.15, p=.05$ ]. In contrast, reaction times for the quasi-aesthetic object were undistinguished across the three modes [mean = 4.54-5.59; $t(37-38) \leq 1.27, p>.05]$.

Consequently, the overall difference between the senses on latency was due to the contribution of the aesthetic object alone. To the extent that latency represents an early stage of processing, equivalence is more characteristic of relatively ordinary rather than aesthetic objects.

There were no differences between the senses on duration $(F<1)$; however, some differential processing must have occurred during this time period. Since touch was slower than sight on the earlier latency period, for the aesthetic object, equivalence between the two senses on duration implies that touch must have been subsequently faster than vision in order to "catch up." Differential cognitive processing, therefore, must have occurred earlier, sometime during the total duration time period. Had the responses been timed throughout the duration period, we could have better determined when equivalence occurred.

Duration times for the senses did not interact with the type of object used $[F(2,52)=1.21, p>.05]$. However, the aesthetic object across modes did require more time than the quasi-aesthetic object [mean $=59.32$ and $51.55 \mathrm{sec}$, respectively; $F(1,52)=4.08, p<.05$ ]. This difference held when latency was taken into account by subtracting it from duration $[F(1,52)=4.82, p<.05]$. The aesthetic object offered more information than did the less aesthetic object; observers needed additional time to examine it.

Nevertheless, despite more time being needed for examination, the number of responses to the aesthetic object did not differ from those of the quasi-aesthetic object [mean $=7.23$ and 6.72 , respectively; $F(1,57) \leq 2.75$, $p>.05]$; this was true for both sensory modes $(F<1)$. (Both the absolute and relative numbers of responses were used; the latter was determined by dividing the absolute number by duration time). Although the aesthetic object required longer reaction times (duration) than did the quasi-aesthetic object, the same number of things were said about each. More reflection does not necessarily mean more output.

Also, no differences were found between the number of responses evoked by each mode of presentation. The combined mode had more responses than either sight or touch alone (mean $=8.08,6.30$, and 6.55 , respectively). Equivalence between the senses was true for both the absolute number of responses $[F(2,57)=2.46, p>.05]$ and for the relative number $(F<1)$.

A sample of the kinds of things said was examined. The first set of responses was taken from the first 12 subjects who received either a visual or a tactile presentation with each type of stimulus. Of the total number of 80 responses, only 6 (all colors; e.g., "green”) could be considered specific to one particular sensory modality (although one subject named a color for the tactile mode). An additional 2 items ("hard," "heavy"), although ostensibly tactile, were ambiguous in that they could be visual referents as well. Five responses were evaluative or functional (e.g., "unappealing," "used as a vase"'). The remaining $90 \%$ of the responses had a neutral sensory identification (e.g., "ridges," "smooth," "has a hole," "a broken edge," "round," "tall"). Thus, according to this rough estimate, the kinds of responses given to both senses were overwhelmingly equivalent.

Equivalence of input between the senses (the latency measure) was found for only the quasi-aesthetic object. Equivalence, on the face of it, characterized the duration data; however, differential processing must have occurred at some point during this time. Equivalence was unambiguously present during output. Hence, the similarities between the ratings across the senses (see Lindauer et al., 1986) were, at the very least, due to equivalence at the time of responding, and to some degree, to equivalence that was present at earlier stages as well.

\section{REFERENCE}

\footnotetext{
Lindauer, M. S., Stergiou, E. A., \& Penn, D. L. (1986). Seeing and touching aesthetic objects: I. Judgments. Bulletin of the Psychonomic Society, 24, 121-124.
}

(Manuscript received for publication February 6, 1986.) 